\title{
Device Software Verification Evaluation Method
}

National Cancer Institute

\section{Source}

National Cancer Institute. Device Software Verification Evaluation Method. NCI

Thesaurus. Code C91996.

Means of assessing a computer program's authenticity, accuracy and uniformity to ensure that it meets its intended specifications. 